\title{
$<$ Chandra: Ten Years of Amazing Science with a Great Observatory.>
}

\author{
$<$ Martin C. Weisskopf $>$ \\ <Space Sciences Department, VP62, NASA/MSFC, Alabama, 35812>
}

\begin{abstract}
We review briefly review the history of the development of the Chandra X-Ray Observatory, highlighting certain details that many attendees of this Conference might not be aware of. We then present a selection of scientific highlights of the first 10 years of this remarkable and unique mission..
\end{abstract}

Keywords: <X-ray Astronomy>

PACS: $\langle 95>$

\section{<INTRODUCTION AND HISTORY>}

It is sobering that there are scientists at this conference who were not born when the Observatory was first conceived. The concept was first articulated in a research proposal that Riccardo Giacconi colleagues wrote in 1963 only 9 months after their discovery of Sco X-1, the first extra-solar X-Ray source, and what came to be known as the "diffuse X-Ray background". The centerpiece of this insightful proposal was a $\approx 1$ meter diameter, 10-meter focal length, grazing-incidence X-Ray telescope with effective area and angular resolution sized to determine the nature of the unresolved background.

In 1976 Riccardo and Co-Principal Investigator Harvey Tananbaum submitted an unsolicited proposal "For the Study of the 1.2 Meter X-Ray Telescope National Observatory" which required arcsecond-scale angular resolution to meet the proposed scientific objectives. The proposal drew sufficient attention at NASA HQ to cause it to initiate a competition amongst the NASA Centers establishing where such a mission might best be accomplished. Marshall teamed with the Smithsonian Astrophysical Observatory (SAO) in vying for, and winning, the management of the mission which, shortly after the assignment, was named the Advanced X-ray Astrophysics Facility (AXAF).

The first AXAF Science Working Group (SWG) for which I served as Vice Chair was chaired by Riccardo, also included: A. Opp (NASA HQ - Ex Officio), E. Boldt, S. Bowyer, G. Clark, A. Davidsen, G. Garmire, B. Kraushaar, R. Novick, K. Pounds, S. Shulman, H. Tananbaum, J. Truemper and A. Walker. It was not until 1983 that the Announcement of Opportunity for the first set of instrumentation was released. AXAF was envisioned as being serviceable at that time. Experiment selection took place in 1985 and a new science working group was formed whose members are pictured in Figure 1.

Because of space limitations I am unable to discuss the years between project initiation and launch in 1999. The interested reader may wish to examine a paper and 


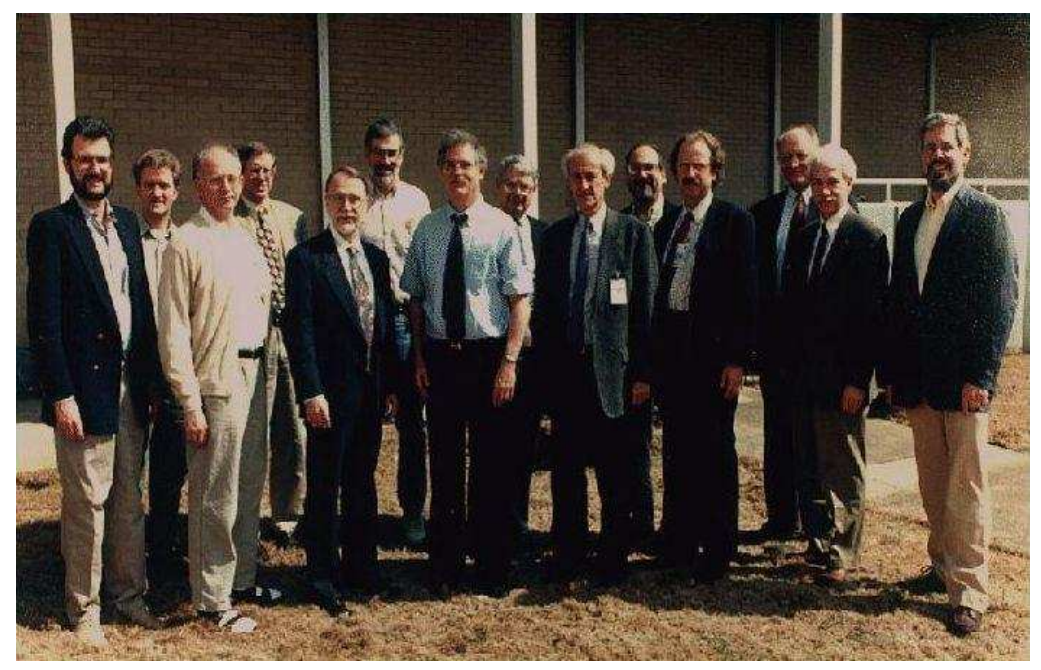

FIGURE 1. The second Science Working Group. Left to right: A. Wilson (Interdisciplinary Scientist - IDS), A. Fabian (IDS), J. Linsky (IDS), H. Tananbaum (head of the SAO mission support team and ultimately Director of the Chandra X-ray Center), A. Bunner (ex officio), S. Holt (Instrument Principal Investigator - IPI), M. Weisskopf (Project Scientist and Chairman), R. Giacconi (IDS), A. Brinkman (IPI), S. Murray (IPI), G. Garmire (IPI), L. van Speybroeck (Telescope Scientist), C. Canizares (IPI), and R. Mushotzky (IDS)

presentation that I made at a recent Chandra Symposium ${ }^{1}$ and also the book by Wallace and Karen Tucker [13]. Some highlights of this time period are listed in Table 1.

In the spring of 1999 the ground-calibrated Observatory was delivered to the Kennedy Space flight Center for integration with the Inertial Upper Stage (IUS) and then into the cargo bay of the Space Shuttle Columbia. This was the heaviest payload ever launched by the Shuttle system.

The activities surrounding the launch had an added attraction as the Commander of Columbia, Eileen Collins, was the first female to hold such a position. Hillary Clinton, the First Lady at the time, paid a visit and the singer Judy Collins (no relation) composed and sang a song dedicated to the astronauts. The attention to Eileen minimized the number of times we were asked if this telescope would have problems such as the Hubble Space telescope experienced initially. Other notables included the widow of Professor Subramanyan Chandrasekhar after whom the Observatory is so aptly named. The male model Fabio attended being under the false assumption that he had been invited by the astronaut Cady Coleman. The invitation had been jokingly issued in her name by her fellow crew members; Eileen, Jeffrey S. Ashby, Steven A. Hawley, and Michel Tognini.

It took two aborted attempts on the mornings of July 20, 22, before the Observatory was successfully launched into its initial orbit on the morning of July 23 . The launch was challenging, due to both a hydrogen leak and an electrical short. Then, after deployment from Columbia, we were concerned about the successful operation of the IUS. Although the IUS had an excellent track record, a previous IUS had failed, albeit for reasons that

\footnotetext{
${ }^{1}$ http://cxc.harvard.edu/ChandraDecade/
} 
TABLE 1. Table 1: Certain Historical Milestones in the development of Chandra

\begin{tabular}{ll}
\hline Time & Event \\
\hline 1976 & Unsolicited proposal leads to selection of MSFC \& SAO to develop the project. \\
\hline 1978 & Launch of the Einstein Observatory, the forerunner to Chandra. \\
\hline 1980 & $\begin{array}{l}\text { Release of the Decadal Survey report "Astronomy and Astrophysics for the 1980's" } \\
\text { which recommended AXAF as the number-1 priority for large, spaced-based missions. }\end{array}$ \\
\hline 1983 & Release of the Announcement of Opportunity for Scientific Investigations. \\
\hline 1985 & $\begin{array}{l}\text { Selection of the Instrument PIs, the IDSs, the Telescope Scientist and formation } \\
\text { of the second SWG. }\end{array}$ \\
\hline 1980 s & $\begin{array}{l}\text { Development and X-Ray testing of the Technology Mirror Assembly comprised of a single } \\
\text { paraboloid-hyperboloid pair and a 2/3-scaled version of the most challenging (innermost) } \\
\text { AXAF mirrors. At 2/3 scale, the focal length was 6-m which allowed for testing in the } \\
\text { existing X-ray test Facility at MSFC, built to calibrate the Einstein X-Ray optics. }\end{array}$ \\
\hline 1991 & $\begin{array}{l}\text { Completion of the initial phase of the expanded X-Ray Test Facility (XRCF) at MSFC. } \\
\text { The XRCF provided x-ray sources, filters, and monochrometers at a distance of 525-m. } \\
\text { and a large thermal vacuum chamber accommodated the Chandra 10-m focal length. }\end{array}$ \\
\hline 1992 & Low-earth orbit and thus servicing abandoned. \\
\hline 1999 & Chandra successfully launched by Columbia and then boosted to its present orbit. \\
\hline
\end{tabular}

were understood. In fact, the IUS placed the Observatory near its final orbit after which followed days of activation, checkout and use of Chandra's internal propulsion system to achieve the final orbit. Perhaps the event which was most intense was the opening of the door to the workhorse instrument aboard Chandra, the Advanced CCD Imaging Spectrometer (ACIS) comprised of 10 CCDs arranged in two arrays. (See [17] for a complete description of the hardware.) The ACIS door opening had failed during ground test and the root cause was never conclusively identified. Because of this, a number of changes and new procedures were established to substantially enhance the probability that the door would open, which, indeed, it did.

The first image taken when the last of several other doors between the detector and outer space was opened included a bright AGN named Leon X-1 [18] in honor of the Telescope Scientist. The ubiquity of AGN in X-ray images was not a surprise, but a forerunner of the fabulous discoveries that Chandra was about to make. Moreover, even the raw (no focus adjustment, no aspect correction) ACIS image of Leon X-1 showed us that no mishap — such as a telescope misalignment - had taken place during the launch. The next tasks in the commissioning were to test and activate the aspect system and to determine the best focus. Our target for these activities was a bright AGN, selected because we wanted a bright point source. Figure 2 shows the image of the bright AGN which, unexpectedly, unrevealed the presence of an X-Ray jet [11]. Even a Chandra "test image" produced a fruitful scientific result. Such discoveries were to be the rule rather than the exception. Perhaps one of Chandra's most famous images (Figure 3), that of the Crab nebula and its pulsar [16] is another case in point. 


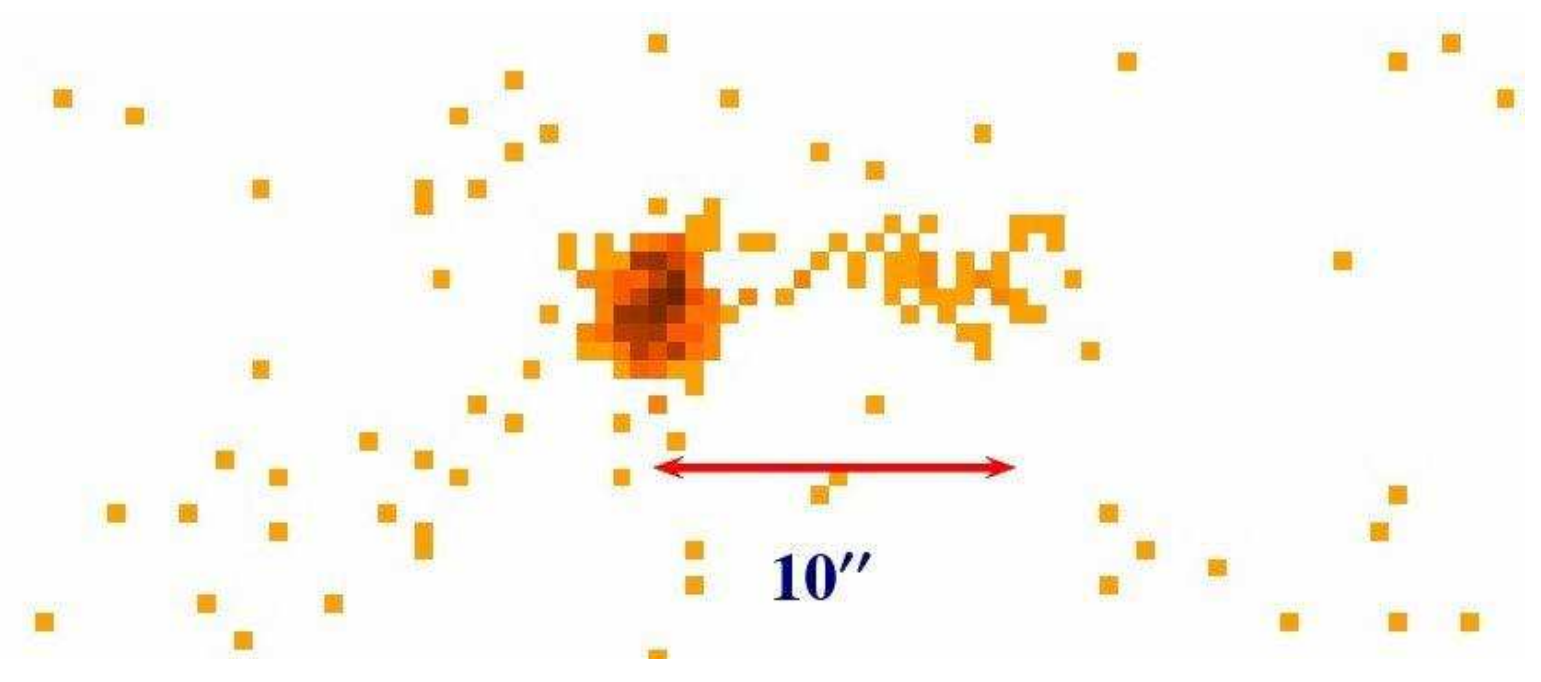

FIGURE 2. Image of Parkes 0637-752 and its X-ray jet.

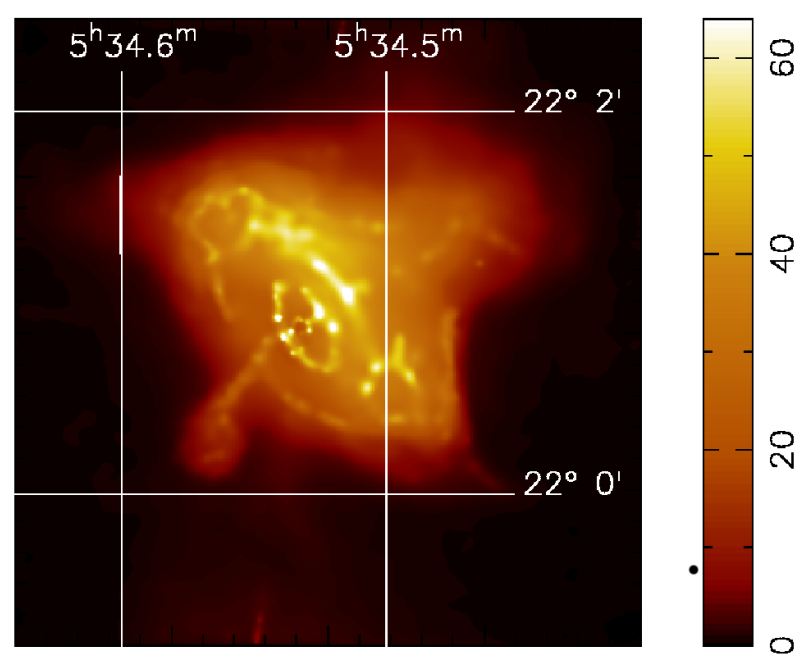

FIGURE 3. The first Chandra image of the Crab Nebula and its pulsar (smoothed) showing rings and jets around the neutron star.

\section{SCIENTIFIC RESULTS}

Now (2009) there have been slightly over 10 years of outstandingly successful scientific research accomplished with this truly Great Observatory. With its vast improvement in spectral resolution and sensitivity over prior missions, and its still unique and superb angular resolution, Chandra observations have served to clarify our understanding of known problems such as the nature of the unresolved X-ray background [4], as well as fostering new areas of research into topics as diverse as comets and dark matter.

Astrophysical insights have been obtained for solar system objects (E.g. [2]), and one now understands that the main emission mechanism for comets is charge exchange. Several planets in the solar system have been observed with Chandra producing discoveries 


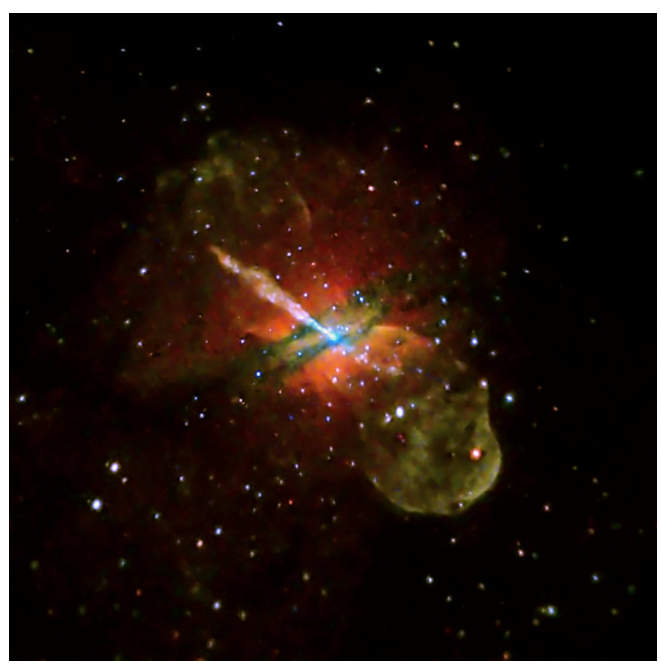

FIGURE 4. Chandra image of Centaurus A, the nearest galaxy with an active SMBH. The prominent jet extends $4 \mathrm{kpc}$ to the $\mathrm{NE}$, with a shorter counterjet in the opposite direction. (NASA/CXC/CfA/ and R.Kraft)

such as the time-variable X-ray flux from Jupiter that originates, not from the UV auroral zone as had been expected, but from higher latitudes near Jupiter's pole - i.e. regions that map to the outer boundary of Jupiter's magnetosphere (E.g. [2]).

Chandra has also collected a wealth of data on the nature of X-ray emission from stars of all ages (E.g. [19], [7]) Considering more distant objects, Chandra (as well as XMMNewton) has been used to discover hundreds of supermassive black holes (SMBH) at the centers of galaxies and in many cases resolved and studied their jets (e.g. Figure 4). High-resolution spectra of SMBH have also provided insight into the accretion process that powers the activity (e.g. [14], [9]). X-ray and radio data demonstrated convincingly that SHMB and intense bursts of star formation can affect the SMBH environment on scales of tens of thousands of parsecs (see the discussion in [5]). Among the most important findings is the strong cosmological evolution in the X-ray luminosity of AGNs [8], [4], [12], and [20].

Chandra has also made profound contributions to the study of Dark Matter and Dark Energy. Figure 5 shows the composite Chandra and optical image of the "bullet" cluster, 1E0657-56, which clearly shows the separation of dark and normal matter in this "cosmic collision" [6]. Finally, galaxy cluster surveys with Chandra have already provided a distance-ladder-independent measure of the Hubble constant [3] and two independent measures of the accelerated expansion of the universe [1], [15].

The interested reader may find more highlights of Chandra in the recent review [10].

\section{ACKNOWLEDGMENTS}

I wish to acknowledge the thousands of scientists throughout the world that have contribute to the success of this great observatory with a special debt of gratitude to Harvey Tanabaum and Steve O'Dell. 


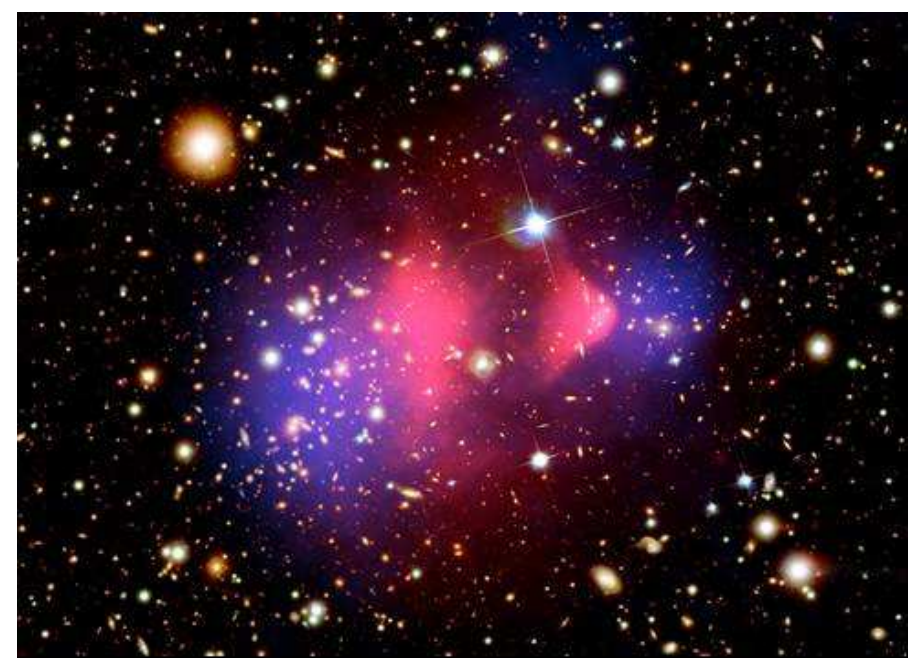

FIGURE 5. Composite image of 1E0657-56. The X-ray emission (pink) constitutes most of the baryonic matter. The optical data (orange and white), from Magellan and the Hubble Space Telescope and shows the galaxies. The blue areas, determined from gravitational lensing, indicate where most of the mass of the cluster lies. (Credit: NASA/CXC Clowe et al. [6].

\section{REFERENCES}

1. S. W. Allen et al. Improved constraints on dark energy from Chandra X-ray observations of the largest relaxed galaxy clusters. Mon. Not. R. Astron. Soc. 383, 879-896 (2008).

2. A. Bhardwaj et al.Planetary and Space Science 55, 1135-1189 (2007) and references cited therein.

3. M. Bonamente, et al. Astrophys. J. 647, 25-54 (2006).,

4. W. N. Brandt and G. Hasinger Annual Review of Astronomy \& Astrophysics 43, 827-859 (2005) and references cited therein.

5. A. Cattaneo et al. Nature 460, 213-219 (2009).

6. D. Clowe et al. Astrophys. J. 648 L109-L113 (2006).

7. M. Guedel, M. and Y. Naze, The Astron. \& Astrophys. Rev. 17, 309-408 (2009)

8. G. Hasinger, T. Miyaji, and M. Schmidt Astron.\& Astrophys. 441, 417-434 (2005).

9. J. M. Miller et al. Astrophys. J. 697, 900-912 (2009).

10. M. Santos-Lleo et al. Nature 462, 997-1004 (2009).

11. D. A. Schwartz et al. The Astrophysical Journal 540, 69-72 (2000).

12. J. D. Silverman et al.Astrophys. J. 679, 118-139 (2008).

13. W. Tucker and K. Tucker, "Revealing the Universe: The Making of the Chandra X-ray Observatory", Harvard University Press, Cambridge, Massachusetts USA (2001).

14. T. J. Turner and L. Miller The Astronomy and Astrophysics Review 17, 47-104 (2009).

15. A. Vikhlinin et al. Astrophys. J. 692,1060-1074 (2009).

16. M. C. Weisskopf, et al. The Astrophysical Journal 536, L81-L84 (2000).

17. M. C. Weisskopf, et al. Publ. Astron. Soc. Pacif. 114, 1-24 (2002)

18. M. C. Weisskopf et al. The Astrophysical Journal 637, 682-692 (2006).

19. S. J. Wolk et al. Astrophys. J. Supp. 160, $423-449$ (2005).

20. B. Yencho, B. et al. Astrophys. J. 698, 380-396 (2009). 